\title{
Comments on Anagnostis et al.: De novo autoimmune hepatitis associated with PTH(1-34) and PTH(1-84) administration for severe osteoporosis in a liver transplant patient
}

\author{
I. Aguilera • A. Nuñez-Roldan
}

Received: 19 January 2012 / Accepted: 26 January 2012 / Published online: 18 February 2012

(C) International Osteoporosis Foundation and National Osteoporosis Foundation 2012

\section{Dear Editor,}

In their recent paper, Anagnostis et al. [1] described a rare case of association of parathyroid hormone (PTH) 1-34 and 1-84 treatment with de novo autoimmune hepatitis (AIH) after liver transplantation (LT). They excluded common causes of liver dysfunction such as viral infections or medication-induced hepatotoxicity. The biopsy findings were compatible with features stated for de novo $\mathrm{AIH}$, and the patient had a good response to methylprednisolone treatment. The authors tested serum samples (they did not say how many or the exact time points after LT) for the common antibodies associated with $\mathrm{AIH}$ and found antinuclear antibodies at 1/160 titer. However, they did not study antiglutathione S-transferase theta 1 (GSTT1) antibodies due to test unavailability.

GSTT1 is a phase II cytosolic enzyme involved in detoxification processes, highly expressed in the liver and kidney. Antibodies against this protein were first described in null GSTT1 patients receiving a graft from a GSTT1-positive donor in patients that developed de novo autoimmune hepatitis after LT [2]. Characterization of the target antigen clearly as a donor antigen led the authors to modify the term auto- for alloimmune or simply immune hepatitis (IH). In addition, the authors demonstrated that the mismatch per se constitutes a risk factor for de novo IH in a study performed

A reply to this comment is available at doi 10.1007/s00198-012-1927-8.

\section{Aguilera $(\varangle) \cdot$ A. Nuñez-Roldan}

Immunology Service, University Hospital Virgen del Rocío

Sevilla/Instituto de Biomedicina de Sevilla (IBIS),

Avda. Manuel Siurot s/n,

41013 Sevilla, Spain

e-mail: iaguilera-ibis@us.es in a large cohort of LT patients [3]. These results were confirmed several years later by other group [4].

We consider that the study by Anagnostis et al. presents interesting data on the alteration of the levels of hepatic enzymes during the course of post-transplant follow-up coinciding with initiation or discontinuation of PTH and could open a new line of research about an alternative pathway leading to de novo IH, distinct from the GSTT1 system. Unfortunately, this remains to be clarified since this report lacks important information concerning data on GSTT1 donor/recipient mismatch as well as anti-GSTT1 antibodies.

Besides that, we have some concerns about the study presentation. Some references are misplaced in the "Introduction" and "Discussion" sections, and other key references have been omitted, such as the ones mentioned in this letter.

\section{References}

1. Anagnostis P, Efstathiadou ZA, Akriviadis E, Hytiroglou P, Kita M (2011) De novo autoimmune hepatitis associated with PTH(1-34) and PTH(1-84) administration for severe osteoporosis in a liver transplant patient. Osteoporos Int. doi:10.1007/s00198-011-1848-y

2. Aguilera I, Wichmann I, Sousa JM, Bernardos A, Franco E, GarciaLozano JR, Nuñez-Roldan A (2001) Antibodies against glutathione S-transferase T1 (GSTT1) in patients with de novo immune hepatitis following liver transplantation. Clin Exp Immunol 126:535-539

3. Aguilera I, Sousa JM, Gavilan F, Bernardos A, Wichmann I, NuñezRoldan A (2004) Glutathione S-transferase T1 mismatch constitutes a risk factor for de novo immune hepatitis after liver transplantation. Liver Transpl 10(9):1166-1172

4. Rodriguez-Mahou M, Salcedo M, Fernandez-Cruz E, Tiscar JL, Bañares R, Clemente G et al (2007) Antibodies against glutathione S-transferase T1 (GSTT1) in patients with GSTT1 null genotype as prognostic marker: long-term follow-up after liver transplantation. Transplantation 83(8):1126-1129 